\title{
DOS ESPECIES NUEVAS DE RHABDEPYRIS (HYMENOPTERA, BETHYLIDAE) DE COLOMBIA
}

\author{
Juan Manuel Vargas-Rojas ${ }^{1}$
}

\begin{abstract}
TWO NEW SPECIES OF RHABDEPYRIS (HYMENOPTERA, BETHYLIDAE) FROM COLOMBIA. Rhabdepyris (Chlorepyris) humboldti sp. nov. and $R$. (C.) tarapachensis sp. nov. from Colombia are described and illustrated. These species are specially peculiar by having pectinate antennae, which is the first report of this character for the genus.
\end{abstract}

KEYWORDS. Bethylidae, Rhabdepyris, Hymenoptera, pectinate antennae, Neotropical.

\section{INTRODUCCION}

Rhabdepyris Kieffer, 1904 es el género, dentro de Epyrini, con distribución en todas las regiones biogeográficas del planeta; con cerca de 110 especies a nivel mundial y con una particular riqueza en el Neotrópico donde se conocen aproximadamente 50 especies (AzEVEDo, 1999). Evans (1965) revisó el género donde describió 28 especies; Azevedo (1992 a, b; 1993; 1999) describió especies, de los subgéneros Rhabdepyris, Chlorepyris y Trichotepyris. El subgénero Chlorepyris reúne 3 grupos de especies, lobatofrons, muscarius y viridissimus (Evans, 1965), 22 especies neotropicales y se caracteriza por presentar coloraciones verde o azul metalizado, talla moderadamente grande, ojos glabros, tercer segmento antenal bien diferenciado del cuarto, pronoto sin surco foveolado paralelo a la margen posterior, tendencia del surco escutelar a ensancharse en fosos en cada extremo y reducirse medialmente a una delgada línea.

Para Evans (1964), las especies de Calyozina Enderlein, 1912 podrían ser meramente machos de diversos linajes especializados de Epyris Westwood, 1832. Evans (1978) comenta que existen varias especies neotropicales de Bethylidae, relacionadas con Epyris donde los machos presentan antenas pectinadas. KROMBEIN (1992) revisó los géneros de Epyrinae con antena pectinada conocidos, determinando 4 sinonimias genéricas y 5 específicas; aunque en dicho trabajo se hace un profundo reconocimiento

1. Fundación Nova Hylaea, Apartado 3567, Santafé de Bogotá D.C., Colombia. (juan_vargas@starmedia.com). 
de los géneros mas representativos de la característica antena pectinada y una importante redefinicion del grupo staphylinoides del género Epyris, en su opinión la antena pectinada es una rareza entre los himenópteros aculeados que ocasionó una explosión de nombres genéricos basada en una característica, de variación intragenérica, presente solo en el macho. Son muy pocos los ejemplares que han sido utilizados para la descripción de especies y géneros, además, aún falta revisar otros grupos genéricos que pueden dar luces sobre el valor taxonómico de este carácter y su verdadero alcance dentro de la discriminación de grupos naturales pues, hasta donde se puede apreciar, la aparición del carácter antena pectinada en Rhabdepyris refuerza la condición homoplásica de dicho carácter dentro de Epyrini.

AzEVEDo (1996) observó antenas pectinadas en machos de Rhabdepyris del Brasil, pero hasta la fecha, no hay dentro de Rhabdepyris especies descritas con antenas pectinadas. Se describen dos especies nuevas, con antenas pectinadas, de Rhabdepyris subgénero Chlorepyris.

El material estudiado pertenece al Instituto de Investigación de Recursos Biológicos "Alexander Von Humboldt" (IAVH) Colombia (Fernando Fernández). Las abreviaciones se ajustan en su mayoría a EvANs (1964): HE, altura de los ojos medida en vista lateral; LB, longitud del cuerpo; LFW, longitud del ala anterior; LH, longitud de la cabeza desde la margen medial del clípeo hasta el punto medio del vertex; OOL, línea oceloocular, mínima distancia entre el ocelo posterior y la margen interna del ojo; VOL, línea del ojo al vertex; WF, ancho de la frente (= mínima distancia interocular); WH, ancho de la cabeza incluyendo los ojos; WOT, ancho del triángulo ocelar incluyendo los ocelos posteriores. La longitud del disco propodeal, del mesoscuto y del disco pronotal es tomada a lo largo de la línea media. La nomenclatura referente a la textura del tegumento es de EADY (1968) y el formato de descripción sigue a AzEvEDo (1992a) con pocas modificaciones.

\section{Rhabdepyris (Chlorepyris) humboldti sp. nov.}

(Figs. 1-10)

Holotipo macho, COLOMBIA, Bolívar, Zambrano (Hacienda Monterrey), IV.1993, F. Fernández col. (IAVH), trampa Malaise.

Etimología. Homenaje al investigador Alexander Von Humboldt y reconocimiento al Instituto de Investigaciones Biológicas IAVH que lleva su nombre.

Macho. Cabeza azul metalizado oscuro; tórax verde metalizado oscuro con reflejos azul oscuro; región apical del gaster y genitalia amarillo rojizo, gaster restante rojo oscuro a negro con reflejos azul oscuro; mandíbulas negras en su tercio basal, porción restante amarillo rojizo, dientes y borde interno de las mismas rojo oscuro; escapo antenal rojo oscuro brillante con un delgado anillo apical amarillo rojizo, flagelo amarillo rojizo opaco con setas claras, coxas negras con sobretonos rojos; fémur, tibia y tarso amarillo rojizo brillante; alas subhialinas con setulas oscuras, venación marrón amarillento; LB 7,5 mm; LFW 4,0 mm.

Cabeza (figs. 1-3): mandíbula con 5 dientes, diente ventral grande, segundo ligeramente más pequeño, los tres dorsales, mucho más pequeños que el primero y redondeados (fig. 3). Clípeo corto, ampliamente subangulado y con una carena media levemente cóncava en perfil y conectando con la región anterior de la frente; coriácea y 


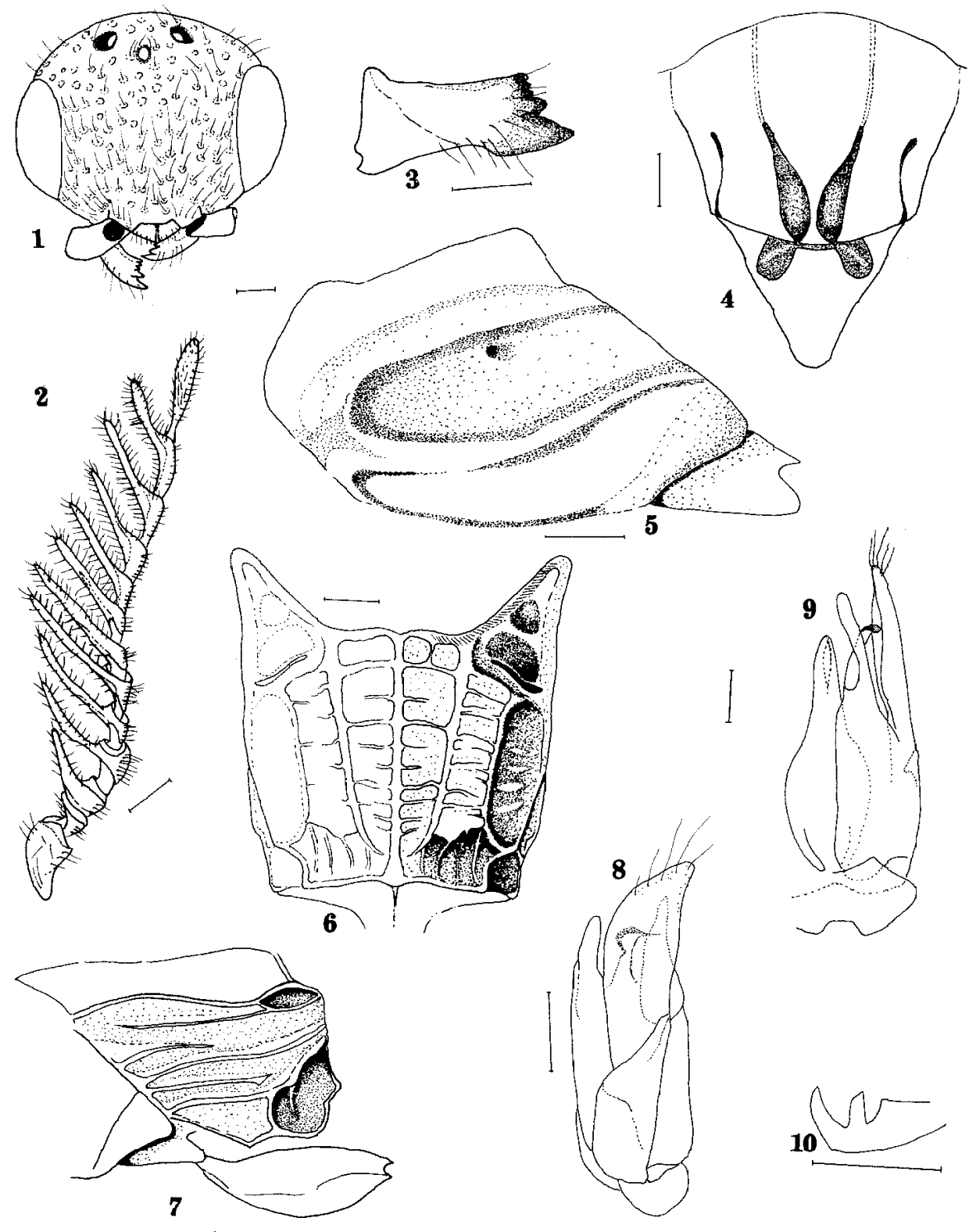

Figs. 1-10. Rhabdepyris (Chlorepyris) humboldti, sp. nov. 1, cabeza, dorsal; 2, antena; 3, mandíbula, frontal; 4, mesonoto, dorsal; 5, mesopleura, lateral; 6, propodeo, dorsal; 7, propodeo, lateral; 8, genitalia, lateral; 9, genitalia, ventral; 10, uña tarsal. Escalas: 0,21 mm, figs. 1-9; 0,10 mm, fig. 10. 
brillante, puncturas aglomeradas sin un patrón definido (fig. 1). Segmentos antenales III-XII con un proceso delgado y peludo cada uno, procesos más largos en VI y VII, 4 x tan largos como su segmento, segmento apical algo clavado; longitud proporcional de los primeros 4 segmentos antenales es 13:5:4:7 (fig. 2). WH 1,18 x LH; WF 1,53 x HE y 0,72 x WH; VOL 0,4 x HE; vertex redondeado; WOT 2 x la altura del triángulo ocelar y aproximadamente igual a la longitud de la línea ocelo-ocular, OOL 1,0 x WOT, ángulo del triángulo ocelar obtuso (fig.1).

Mesosoma (figs. 4-7, 10): dorso torácico coriáceo y brillante; pronoto con puncturaciones más pequeñas y menos aglomeradas que en la cabeza; ancho del disco pronotal 0,59 x su longitud y $0,47 \mathrm{x}$ el largo del mesoscuto, margen posterior del disco pronotal con una línea de foveas pequeñas sobre una banda lisa y pulida; notaulo no alcanzando la margen anterior pero conectado con ésta por líneas pulidas débilmente impresas, separado posteriormente de la margen por un surco angosto, surcos parapsidales ocupando poco más de la mitad de la longitud total del mesoscuto, fosos escutelares subcirculares separados por poco más que sus propios diámetros, conectados por un estrecho surco de igual profundidad (fig. 4). Mesopleura con una fovea oblonga abierta posteriormente, y una fovea ventrolateral más grande (fig. 5). Disco propodeal 1,63 x tan ancho como largo, con 3 carenas discales, discal mediana completa, las dos discales paramedianas convergen posteriormente y casi completas, 2 carenas sublaterales y 2 laterales, espacio entre carenas con estrías transversales (fig. 6). Lado del propodeo con 6 crestas gruesas, pulido y brillante (fig. 7). Uñas tarsales trifidas (fig. 10).

Gaster: no peciolado, tergitos lisos y brillantes, I y II dorsolateralmente glabros con algo de pilosidad lateral, tergitos III al VII con borde anterior coriáceo, pilosidad dispersa y escasa, longitud del gaster 1,3 x la longitud del mesosoma. Genitalia (figs. 8, 9): parámero de ápice truncado, inclinado y ancho en vista lateral, margen ventral ligeramente cóncava en la región apical, convexa en la región media y cóncava en la región basal lo cual le confiere un aspecto sinuoso lateralmente, margen dorsal convexa en la parte apical y recta en la basal, márgenes dorsal y ventral convergiendo basalmente (fig. 8); volsella con el cuspis más corto que el parámero, digitus mas corto que el cuspis, aedeago en forma de botella con la base redondeada, ligeramente mas bajo que el digitus y paralelo al parámero, apodemas convergentes apicalmente que no sobrepasan el borde ventral del anillo basal (fig. 9).

\section{Rhabdepyris (Chlorepyris) tarapachensis sp. nov.}

(Figs. 11-20)

Holotipo macho, COLOMBIA, Amazonas, Km 22, vía Tarapacá (NE de Leticia), 29.VIII.1997, D. Campos col. (IAVH), red entomológica.

Etimología: alusivo a Tarapacá, nombre de una localidad cercana al sitio de captura del espécimen tipo.

Macho. Cabeza verde metalizado; mesosoma verde oscuro; gaster negro; palpos marrón, mandíbulas marrón rojizo; escapo negro, pedicelo marrón oscuro con setas oscuras; coxas negras; fémures anteriores amarillo marrón, medios y posteriores marrón, trocanteres marrones, tibias y tarsos amarillos; alas subhialinas con venas marrón; propodeo relativamente glabro respecto al resto del cuerpo, gaster cubierto por setas oscuras; LB 6,03 mm; LFW 3,0 mm. 

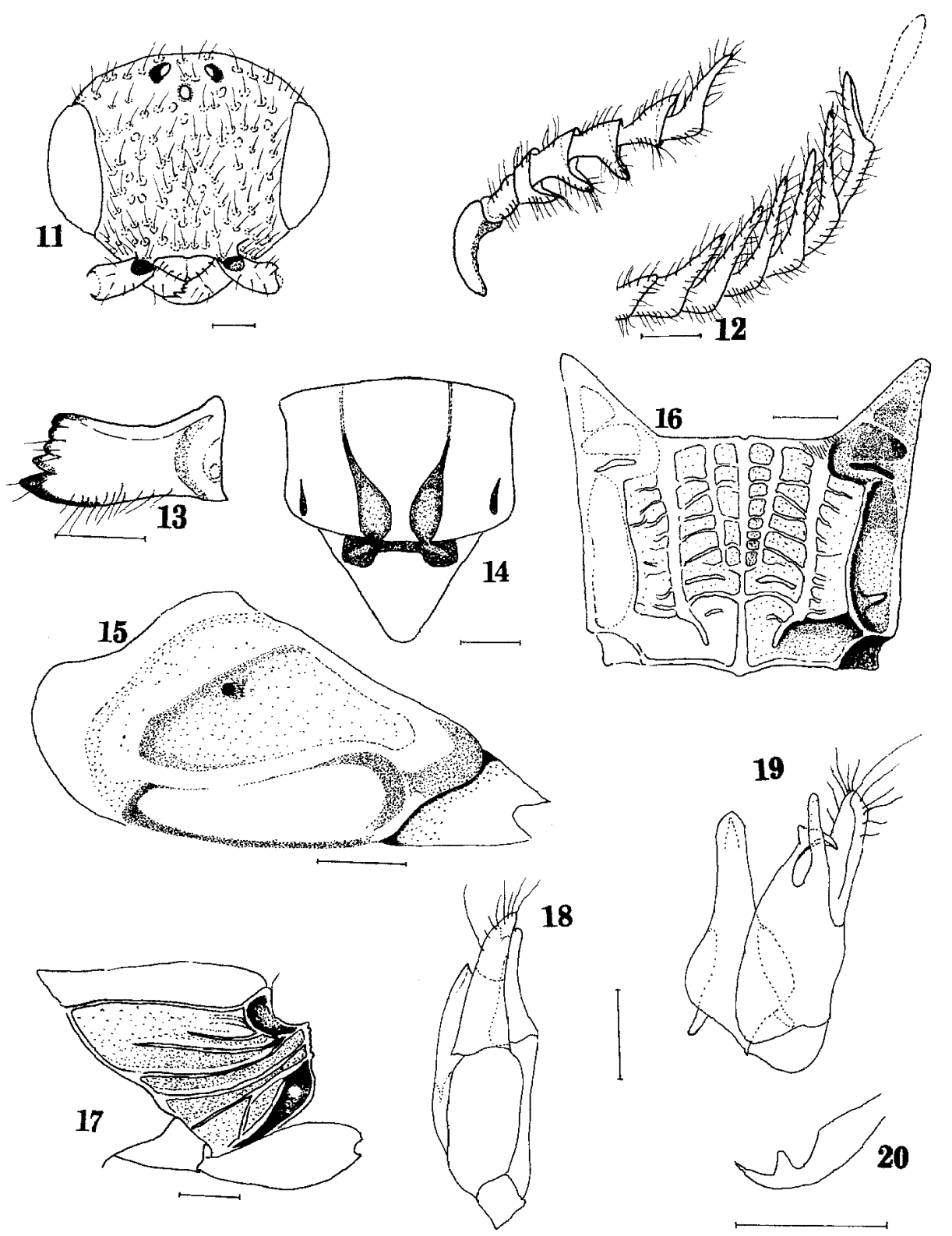

Figs. 11-20. Rhabdepyris (Chlorepyris) tarapachensis, sp. nov. 11, cabeza, dorsal; 12, antena; 13, mandíbula, frontal; 14, mesonoto, dorsal; 15, mesopleura, lateral; 16, propodeo, dorsal; 17, propodeo, lateral; 18, genitalia, lateral; 19, genitalia, ventral; 20, uña tarsal. Escalas: 0,21 mm, figs. 11- 19; 0,07 mm, fig. 20. 
Cabeza (figs. 11-13): mandíbulas con 5 dientes, el ventral más grande y agudo, los demás redondeados y decrecen hacia el borde interior (fig. 13). Clípeo obtusamente angulado, con una carena media recta en perfil, bien distinguible y terminando en una pequeña carena transversa que se extiende entre los fosos antenales; débilmente coriácea, puncturas aglomeradas sin un patrón definido (fig. 11). Segmentos antenales III-V y VII-XII con un proceso delgado y setoso; proceso VI, aunque ensanchado, sin proceso distinguible y separa dos grupos de segmentos con las pectinas dirigidas en sentido casi opuesto; los segmentos VIII, IX, X y el XI con procesos más largos con 2,1 x, 2,2 x, 1,8 x y 1,8 x su segmento; longitud proporcional de los primeros 4 segmentos antenales 12:5:3:6 (fig. 12). WH 0,81 x LH; WF 1,46 x HE y 0,70 x WH; VOL 0,32 x HE, vertex redondeado; WOT 1,43 x altura del triángulo ocelar, OOL 1,0 x WOT, ángulo del triángulo ocelar recto (fig. 11).Mesosoma (fig. 14-17, 20): disco pronotal y mesoscuto coriáceos, escutelo pulido y brillante, dorso puncturado como la cabeza; disco pronotal 1,63 x tan ancho como su longitud y 2,14 x el largo del mesoscuto, notaulo alcanzando la margen posterior del mesoscuto y conectado con la anterior por líneas pulidas débilmente; surcos parapsidales ocupando el $1 / 4$ posterior del mesoscuto y parte cercana a la línea media; fosos escutelares profundos, subcirculares y unidos por un surco estrecho y profundo (fig. 14). Mesopleura con una fovea central grande alargándose atrás, algo curvada, con extremo posterior redondeado dirigiéndose ventralmente y una fovea ventrolateral más grande y menos definida (fig. 15). Disco propodeal $0,71 \mathrm{x}$ tan largo como ancho y con 5 carenas discales, medial completa, las discales mayores cubren casi todo el disco, incompletas y convergentes posteriormente, discales menores $0,5 \mathrm{x}$ tan largas como el disco y convergentes posteriormente, carenas sublaterales curvas, las demás más o menos rectas, espacio entre las carenas lateral y sublateral pulida, más baja que el resto del disco y con estrías transversales, espacios entre las carenas discales y la medial estriados y forman una depresión con respecto a los espacios entre las discales mayores y las sublaterales (fig. 16). Lados del propodeo pulidos y brillantes con 5 estrías longitudinales finas en dirección anteroposterior (fig. 17). Uñas tarsales trifidas (fig. 20).

Gaster: no peciolado, tergitos abdominales lisos y pulidos, I y II glabros, del III al VII poblados de abundantes setas oscuras y con las márgenes anteriores coriáceas, sección transversal circular, longitud del gaster 0,92x longitud del mesosoma. Genitalia (figs. 18, 19): parámero de ápice fino, angosto, margen ventral ligeramente cóncava en la mitad apical y recta en la mitad basal, margen dorsal ligeramente convexa en la mitad apical y recta en la mitad basal, márgenes ventral y dorsal divergiendo basalmente (fig. 18); volsella con el cuspis ligeramente más corto que el parámero, digitus mas corto que el cuspis, aedeago en forma de botella con aristas en la parte basal, ligeramente mas alto que el digitus y no paralelo con respecto al parámero, apodemas divergentes apicalmente que no sobrepasan el borde ventral del anillo basal (fig. 19).

\section{DISCUSIÓN}

Rhabdepyris (C.) humboldti y R. (C.) tarapachensis son muy diferentes en lo que se refiere al grado de pilosidad general, longitud y dirección de las pectinas (forma y grado de desarrollo), número de carenas propodeales, forma de la carena clipeal, 
estructura general de la genitalia (especialmente en forma y longitud de los parámeros), tamaño y disposición de las carenas en el lado del propodeo. Sin embargo, hay uniformidad en caracteres tales como forma del clípeo, esculturación alutacea de casi todo el cuerpo y presencia de puncturaciones en la cabeza y el pronoto principalmente, número de dientes en las uñas tarsales, número y forma de los dientes mandibulares. La ilustración de la antena de $R$.(C.) tarapachensis fue dividida en dos partes para poder observar las pectinas de los segmentos VII a XII. Las especies pertenecen al grupo viridissimus (en el sentido de Evans, 1965) debido a su coloración verde-azul brillante, surco escutelar en forma de fosos pareados conectados por un surco delgado y uñas trifidas; el macho de $R$. humboldti presenta la mayor longitud corporal de todos los machos del grupo; aunque $R$. tarapachensis es de menor tamaño que $R$. humboldti sigue presentando una talla considerable.

Agradecimientos. Al Doctor Celso Oliveira Azevedo, del Departamento de Biología de la Universidade Federal do Espírito Santo, por su orientación y apoyo bibliográfico, a Fernando Fernández Ms. C. (IAVH) por el préstamo del material, a Edgard Palacio y Carlos Sarmiento Ms. C., de la Fundación Nova Hylaea, por sus valiosos aportes y comentarios.

\section{REFERENCIAS BIBLIOGRAFICAS}

AzEvedo, C. O. 1992a. On Rhabdepyris Kieffer (Hymenoptera, Bethylidae) in the area of São Carlos: I. Subgenus Rhabdepyris (Rhabdepyris). Revta bras. Ent., São Paulo, 36(1): 241-244.

. 1992b. Sobre os Rhabdepyris Kieffer (Hymenoptera, Bethylidae) coletados em Piçarras, SC, Brasil. Revta bras. Ent., São Paulo, 36(2): 251-254.

. 1993. On Rhabdepyris Kieffer (Hymenoptera, Bethylidae) in the area of São Carlos, State of São Paulo, Brazil: II. Subgenus Trichotepyris. Revta bras. Ent., São Paulo, 37(2): 305-308.

. 1996. Descrição de uma espécie nova de Calyozina Enderlein (Hymenoptera, Bethylidae) do Brasil. Revta bras. Zool., Curitiba, 13(4): 923-927.

1999. On the Neotropical Rhabdepyris Kieffer (Hymenoptera, Bethylidae) of the subgenus Chlorepyris.

Revta bras. Zool., Curitiba, 16(3): 887-897.

EADY, R. D. 1968. Some Illustrations of microsculpture in the Hymenoptera. Proc. R. ent. Soc. Lond., (A), London, 43 (4-6): 66-72.

Evans, H. E. 1964. A synopsis of the American Bethylidae (Hymenoptera: Aculeata). Bull. Mus. comp. Zool., Cambridge, Mass., 132(1): 1-222.

. 1965. A revision of the genus Rhabdepyris in the Americas (Hymenoptera, Bethylidae). Bull. Mus. comp.

Zool., Cambridge, Mass., 133(2): 67-151.

1978. New Neotropical Calyozina, with key to species (Hymenoptera: Bethylidae). Ent. News, Philadelphia, 1-2(89): 61-62.

Krombein, K. V. 1992. Systematics of the genera of Epyrini with ramose male antennae (Hymenoptera, Bethylidae). Proc. ent. Soc. Wash., Washington, 94(3): 345-360. 